\title{
Recent Approaches in the Treatment of Polycystic Ovary Syndrome: An Update
}

\author{
Shagufta ${ }^{1 *}$, Irshad Ahmad ${ }^{1}$, Noora Ali Nasar ${ }^{2}$ and Maheen Imtiaz ${ }^{2}$ \\ ${ }^{1}$ Department of Mathematics and Natural Sciences, School of Arts and Sciences, American University of Ras Al Khaimah, Ras Al Khaimah, UAE \\ ${ }^{2}$ Department of Biotechnology, School of Arts and Sciences, American University of Ras Al Khaimah, Ras Al Khaimah, UAE
}

${ }^{\star}$ Corresponding author: Shagufta, Department of Mathematics and Natural Sciences, School of Arts and Sciences, American University of Ras Al Khaimah, Ras Al Khaimah, UAE

Received: February 21, 2022; Accepted: February 28, 2022; Published: March 03, 2022

\begin{abstract}
Polycystic Ovary Syndrome (PCOS) is a dominant and complex endocrine disorder present in women worldwide. The characteristic features include anovulation, polycystic ovaries, insulin resistance, menstrual irregularities, and hyperandrogenism-related difficulties. Medical treatment of PCOS focus on symptoms, and several drugs, natural products, and herbal plants are applicable to reduce PCOS-associated symptoms. This review discussed the treatment options for PCOS women, including lifestyle changes, bariatric surgery, and therapy for anovulation, insulin resistance, menstrual dysfunction, and hyperandrogenism-related symptoms. Furthermore, it provides the chemical structure of drugs and natural products exhibiting effectiveness in PCOS treatment.
\end{abstract}

We anticipate that the information provided in this review is beneficial to scientists globally associated with the discovery and development of PCOS treatment in the pharmaceutical industry and academia.

Keywords: Polycystic ovary syndrome (PCOS), Anovulation, Insulin resistance, Menstrual dysfunction, Acne, Alopecia

\section{Introduction}

Polycystic Ovary Syndrome (PCOS) is a common endocrine condition of hormonal imbalance in women, and it is affecting at least $5 \%$ to $10 \%$ of women of reproductive age [1]. Stein and Leventhal first described PCOS in 1953. It is a complicated ovarian disorder characterized by the clinical and biochemical manifestation of hyperandrogenism, ovulatory dysfunction (menstrual disturbances), and polycystic ovaries and one of the most common endocrinopathies in reproductive-age women of the developed world [2]. The main characters of PCOS include excess production of male hormone androgen by the ovaries resulting in anovulatory infertility. Fundamentally, this happens because of the unbalanced release of Luteinizing Hormone (LH) and Follicle-Stimulating Hormone (FSH) from the pituitary gland. The FSH is accountable for follicular development, and increasing the level of the female hormone estrogen and therefore diminution of its level in the bloodstream results in the undeveloped follicles. Several immature follicles dissolve, and the rest of them remain as fluid-filled sac known as cysts, and the name PCOS is derived from these developed cysts in one or both ovaries (Figure 1).

Furthermore, not all PCOS patients develop a cyst, and cyst formation might occur in women with no PCOS condition. PCOS patients mainly build insulin resistance resulting in an increased insulin level, and it is one of the significant symptoms and underlying physiopathological causes of PCOS [3]. High insulin and LH levels result in hyperandrogenemia, which involves excess production of the male hormone androgen in females. It is one of the main reasons for PCOS's clinical complications, including anovulation, abnormal menstrual cycle, and infertility. Furthermore, hyperandrogenism can initiate hirsutism, acne, androgenic alopecia, and metabolic abnormalities such as obesity, insulin resistance, hyperinsulinemia, and dyslipidemia. In many PCOS women, lipid abnormalities, exceptionally high triglyceride, low high-density lipoprotein cholesterol levels, and impaired fibrinolysis have been observed.

PCOS and associated complications physically affect the female body, and the complicated connection between genetics, environment, and hormones instigates mental health problems [4]. Reports suggest

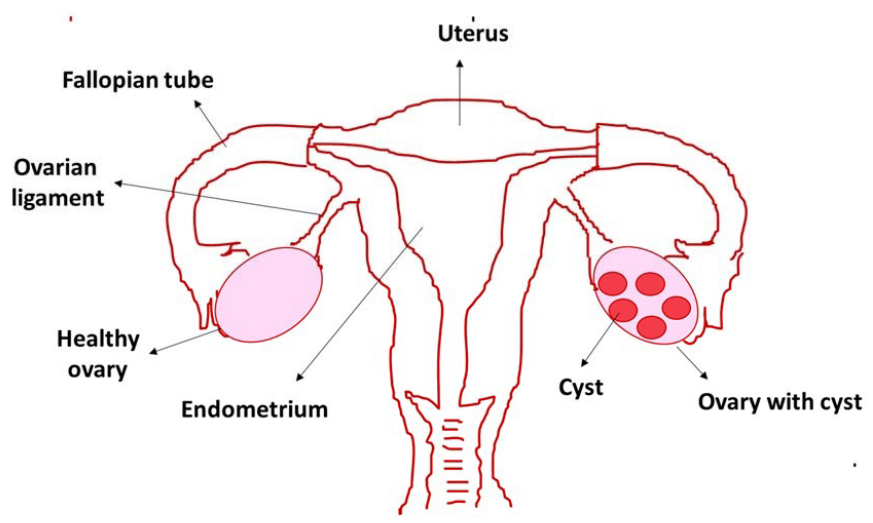

Figure 1: Polycystic Ovary Syndrome (PCOS) in women with cysts in the ovary. 
that PCOS patients are three times presumably suffer from depression, stress, anxiety, and Obsessive-Compulsive Disorder (OCD), bipolar illness, and eating problems. In PCOS women, the depression could be biological or because of distress associated with infertility, abnormal menstrual cycles, acne, body shape, and appearance or external pressure, including demands related to marriage, children, societal attitudes, standards, and culture. In certain cultures, irregular menstrual cycles link to a reduced sense of feminine identity, and therefore women develop feelings of being incomplete. PCOS-related ovulatory dysfunction is responsible for 75 percent of all female infertility cases and severely impacts psychological well-being, including depression in infertile women. Nevertheless, depression scores were still higher in PCOS patients in trials that included only infertile women or omitted all infertile women.

Polycystic ovary syndrome is a metabolic, hormonal, and psychosocial disorder with distinct biopsychosocial aspects and causing severe impacts on the life of PCOS patients. Women must take PCOS symptoms seriously and consult a medical professional or a counselor about any distress they are experiencing, as well as any misconceptions they may have regarding PCOS. The medical practitioner and gynecologist can facilitate the diagnosis and offer proper treatment and advice to manage PCOS. It is advisable to take good care of one's physiological and physical health during PCOS treatment. In this respect, the advice from mental health professionals and psychologists and the lifestyle change are beneficial. Comprehensive treatment of PCOS patients in the early stages will help them overcome emotional stress; a significant complication mostly overlooked in PCOS patients. Early diagnosis and longterm management of PCOS will reduce its associated long-term complications, including metabolic syndrome and cardiovascular diseases, and allow PCOS women to live healthy and happy, active lives [5]. This review article is within the context of our ongoing drug design and discovery research work [6-14]. Here we have compiled the inclusive information related to PCOS, including its symptoms, management, and treatment, focusing on approved drugs. This article is valuable to PCOS patients and doctors, scientists involved in PCOSrelated treatment and research, and anyone interested in acquiring more information on PCOS.

\section{Common Symptoms of PCOS}

PCOS symptoms are present in different ways in women with variations in severity and can change at various stages of women's life. Some women can have only a few symptoms, mild symptoms, or many symptoms with seriousness. In PCOS women, menstrual cycles mostly last more than 35 days, as opposed to the usual 25- to 35-day menstrual cycle duration, and many women have fewer than six periods per year [15]. The women's irregular menstrual cycle leads to the disruption of the ovulation schedule because of the hormonal imbalance. The eggs located in the follicles do not mature and ovulate, resulting in cysts in the ovaries and periods or irregular periods.

Furthermore, because of the inconsistent ovulation or inability to ovulate, the female may have trouble getting pregnant. The bodily mechanisms of normal females are different from PCOS females [16]. The hormone insulin, mainly responsible for converting sugars and starches into energy, is not utilized adequately, resulting in high insulin concentration and insulin resistance in PCOS women. Higher insulin level causes more release of male hormone androgen (hyperandrogenism) that induces weight gain. Hyperandrogenism can result in hirsutism, androgenic alopecia, or female pattern hair loss, specifically from the top of the scalp and forehead. Androgen hormones also induce the sebaceous glands to expand and produce more sebum resulting in acne on the face and body. In addition, acanthosis nigricans is witness as one of the symptoms of PCOS. It is an unsightly harmless skin condition that involves the development of patches of dark or dense skin on particular parts of your body, particularly on the pelvic area, beneath the breasts, armpits, and nape (i.e., back of the neck) is witnesses as one of the symptoms of PCOS. Skin tags are one of the less frequent signs of PCOS, although they do happen. Tiny, flexible lumps or flaps of skin are known as skin tags. PCOS-related skin tags usually develop in the same places as dark skin patches. The long-term effects of PCOS in later life include type 2 diabetes, cardiovascular diseases, and sleep apnoea, a sleeping disorder where breathing is interrupted. For several years, if a woman didn't have periods or had highly irregular periods of less than four periods in a year, they have a greater than usual chance of getting womb lining cancer (endometrial cancer). However, the risk of developing endometrial cancer is still low and reduced by taking period-controlling medications such as the contraceptive pill or an intrauterine system (IUS). The PCOS symptoms also impair women's confidence and self-esteem, and they might experience sadness and mood fluctuation [17].

\section{Treatment Options for PCOS Women}

As such, there is no specific treatment for PCOS. However, its therapy involves symptoms suppression. PCOS treatment is symptombased (Figure 2) and focused on the primary leading illness. Therefore, treatment plan varies from person to person based on the symptoms. Here in this section, we will discuss in detail the various approaches utilized in PCOS treatment.

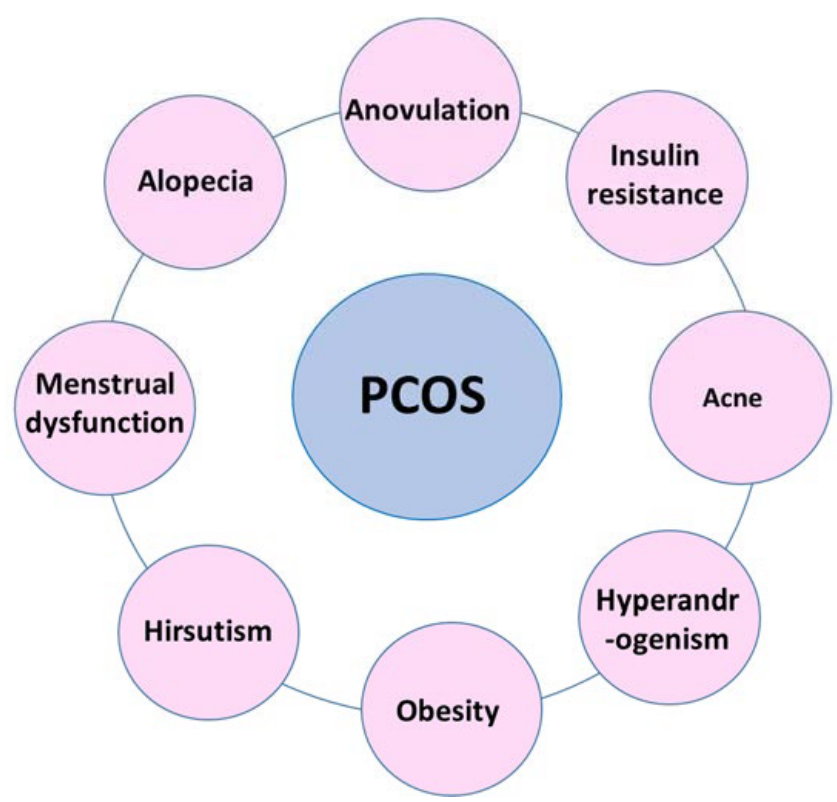

Figure 2: Most common PCOS-associated clinical symptoms. 


\section{Lifestyle Changes}

The primary treatment approaches for women with polycystic ovary syndrome include diet, weight loss, and exercise. Obesity is one of the major issues not only in women but in our society as well. It is responsible for abnormalities in the reproductive system and metabolism, particularly in PCOS women [18]. Around 40$80 \%$ of PCOS patients suffer from obesity-associated anovulation, miscarriage, and late pregnancy complications. Some reports suggest that hyperandrogenism in PCOS women can cause obesity with a high waist/hip ratio independent of the Body Mass Index (BMI) [19]. Weight loss is a first-line treatment in obese PCOS patients with infertility issues. However, it is only recommended in overweight PCOS patients with a BMI of $25-27 \mathrm{~kg} / \mathrm{m}^{2}$. In obese PCOS women, it is observed that weight loss can improves the endocrine profile and circulating androgen and glucose level and increases the ovulation and pregnancy rates.

Additionally, in obese PCOS patients, it is recommended to follow a hypocaloric diet with low carbohydrate intake that can be easily tolerated and maintained [20]. A low-fat diet reduces hyperinsulinemia and improves metabolism. Integration of low glycemic index food in the diet has exhibited improved insulin sensitivity and ovarian function. PCOS women mainly have a higher level of inflammation; therefore, an anti-inflammatory diet is recommended for PCOS women. Regular exercise and a nutritious diet can help maintain the bodyweight of PCOS patients and, therefore, can decrease the risk of severe health conditions, particularly high blood pressure, stroke, sleep apnea, etc. [21]. In addition, exercise mainly improves glucose metabolism and regulates insulin level, lowering testosterone level and helping to battle testosterone-related symptoms such as acne, hair loss, and unusual hair growth on the face. Undoubtedly, exercise is essential for PCOS patients. Still, the exercise program must be designed with the best activity of adequate duration and frequency, based on the patient's degree of obesity and baseline fitness.

\section{Bariatric Surgery}

Recently, bariatric surgery emerged as an alternative approach for weight loss in obese PCOS patients who cannot reduce weight with diet and exercise [22]. However, the perfect bariatric procedure for PCOS is unidentified; the most common approaches include restrictive and combined restrictive, malabsorptive practices, adjustable gastric banding, and the Roux-en-Y gastric bypass. Reports suggest that bariatric surgery in some PCOS women effectively improves ovulation, insulin resistance, hyperandrogenism, and hirsutism. In several obese PCOS patients, a regular menstrual cycle is restored after bariatric surgery [23]. Undoubtedly, bariatric surgery leads to weight loss and therefore results in resumed ovulation and pregnancy. However, they are at higher risk of nutritional deficiency affecting the fetus's nutrition and pregnancy. Reports suggest that women who conceive after bariatric surgery are at more risk for mall-for-gestational-age babies and shorter pregnancies. Although bariatric surgery has its own short and long-term risks, considering its potential benefits, it can be included as part of the treatment in PCOS women.

\section{Therapy for Anovulation (Infertility) in PCOS Women}

Ovulation is a vigorous process that involves highly coordinated endocrine changes with the hypothalamus, pituitary, and ovaries. Anovulation is the lack of ovulation and the most common cause of infertility that affects around $30 \%$ of infertile women. PCOS condition is one of the most commonly encountered reasons for anovulation [24]. In PCOS women, anovulation is described by the better response of some follicles to FSH and $\mathrm{LH}$, multiple follicle development, and the arrest of antral follicles related to the suppression of serum FSH. High levels of LH, androgens, and insulin play a significant part in the anovulation process, enhancing steroidogenesis and stopping follicular growth. Several fertility drugs and medications can normalize ovulation, and we have discussed them in this section.

\section{Selective Estrogen Receptor Modulators}

Selective Estrogen Receptor Modulators (SERMs) are antiestrogens having selectivity to stimulate or inhibit the estrogen receptor of different target tissues and show variation in the ratio of the estrogenic and antiestrogenic at other tissues [25]. Clomiphene (Figure 3) (brand name Clomid and Serophene) is a triphenylethylene-derived nonsteroidal selective estrogen receptor modulator used as an ovulationinducing drug for the last 40 years. It is the first-line treatment in an ovulating woman, including PCOS patients. Clomiphene functions by affecting the hypothalamus and pituitary gland and subsequently increasing $\mathrm{GnRH}, \mathrm{FSH}$, and $\mathrm{LH}$ secretion that leads to follicle (egg) development and ovulation [26]. The pregnancy rate with clomiphene is $30-40 \%$ despite a reasonable ovulation rate of $70-80 \%$, credited to the anti-estrogenic effect of clomiphene on endometrium and cervix. The multiple pregnancy rates after conception with clomiphene is $7.8 \%$, with most twins and triplet or less than $0.9 \%$. With Clomiphene treatment, mainly pregnancy is observed within the first six ovulatory cycles, and the live birth rate ranges from 20 to $40 \%$.

Starting dose for clomiphene is $50 \mathrm{mg} /$ day for five days and then increased to $50 \mathrm{mg} /$ day and a maximum of $150 \mathrm{mg} /$ day in subsequent cycles if the patient remains anovulatory. However, clomiphene usage is associated with side effects including bloating, stomach or pelvic pain, ovarian enlargement, dizziness or headaches, hot flashes, abnormal uterine bleeding, yellow eyes or skin, nervousness, and change in vision or loss in vision possibly because of anti-estrogenic effect in the hypothalamus [27].

Tamoxifen (Figure 3) is another non-steroidal selective estrogen receptor modulator with a triarylethylene skeleton, which is used as an oral ovulatory agent because of its antiestrogenic activity clomiphene. Tamoxifen pregnancy rate $(30-50 \%)$ and ovulation rate $(50-90 \%)$ is better than tamoxifen [28]. Tamoxifen without the involvement of hypothalamic-releasing factors stimulates the ovary and promotes folliculogenesis. Tamoxifen exhibits a minimal anti-estrogenic effect on the cervix and endometrium and enhanced functioning of the corpus luteum; therefore considered an appropriate alternative to Clomiphene in PCOS patients who fail to ovulate with clomiphene or show clomiphene resistance [29]. The side effects of tamoxifen treatment include bone pain, hot flashes, nausea, fatigue, mood swings, depression, headache, and hair thinning. 
<smiles>CCN(CC)CCOc1ccc(C(=C(Cl)c2ccccc2)c2ccccc2)cc1</smiles>

Clomiphene<smiles>N#Cc1ccc(C(c2ccc(C#N)cc2)n2cncn2)cc1</smiles>

Letrozole<smiles>CCC(=C(c1ccccc1)c1ccccc1)c1ccc(OCCN(C)C)cc1</smiles>

Tamoxifene

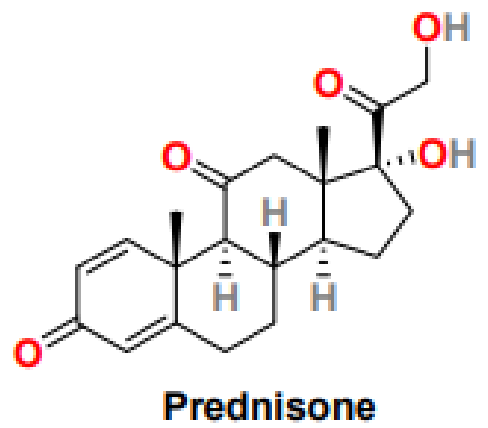<smiles>CC(C)(C#N)c1cc(Cn2cncn2)cc(C(C)(C)C#N)c1</smiles>

Anastrozole

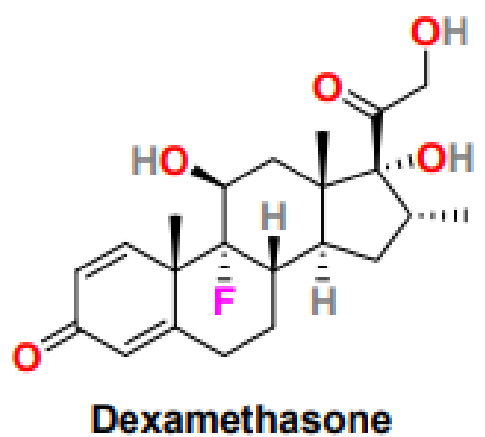

Figure 3: Chemical structure of drugs for the treatment of anovulation in PCOS women.

\section{Aromatase Inhibitors}

Aromatase inhibitors are competitive inhibitors of enzyme aromatase that play a crucial role in the final step of the estrogen biosynthetic pathway. Anastrozole and letrozole (Figure 3) are nonsteroidal aromatase inhibitors with encouraging ovulation-inducing properties [30]. Anastrozole and letrozole are selective, reversible, and highly potent aromatase inhibitors with a half-life of around 45 hours, much better than clomiphene (half-life 5-7 days). Compared to anastrozole, letrozole is studied comprehensively and considered first-line therapy in PCOS women for ovulation induction. Letrozole functions by inhibiting estrogen production in the hypothalamuspituitary axis leading to an upsurge in Gonadotropin-Releasing Hormone $(\mathrm{GnRH})$ and FSH level and subsequently results in enhancing the mature follicle quantity, optimizing ovulation, and increasing the pregnancy rate in PCOS women. The letrozole offers several advantages as an ovulation-inducing agent, including oral administration, shorter half-life, minimal peripheral antiestrogenic effect on the endometrium, higher implantation rate, and stimulating mono follicular growth ovulation, therefore, reducing the rate of multiple pregnancies [31]. Compared to clomiphene, letrozole has exhibited a 50-60\% live birth rate, lower multiple pregnancy rate, higher per cycle and cumulative ovulation rate, and better quality ovulation. Letrozole can effectively induce ovulation in women with the estrogen-sensitive disease, particularly breast cancer, and longterm letrozole treatment is safe and effective in postmenopausal women with early breast cancer. Letrozole in long-term use produces more mature follicles and successful pregnancies than its short-term usage [32]. The most common side effects of letrozole include hot flashes, headaches, dizziness, weakness, bone pain, muscle or joint pain, swelling, and weight gain.

\section{Gonadotropins}

Gonadotropins are hormones secreted from the anterior pituitary gland and act on the gonads to increase the production of sex hormones and stimulate ova production in females. The main gonadotropin hormones are Follicle-Stimulating Hormones (FSH) and Luteinizing Hormones (LH). Exogenous gonadotropins are used as a secondline treatment to induce ovulation in PCOS women who developed resistance to clomiphene [33]. Gonadotropins work by inducing ovulation, maintaining follicle growth, and attaining healthy follicles for fertilization with no peripheral anti- estrogenic effect. Gonadotropins in low doses offer a high ovulation rate, mono follicular development, and lower the risk of Ovarian Hyperstimulation Syndrome (OHSS) and multiple pregnancies [34]. Patients who are unsuccessful in ovulating after clomiphene treatment suffers from severe ovarian abnormality, requiring a higher threshold dose of exogenous FSH. Whereas the patients who are successful in ovulation but unable to conceive after clomiphene intake need a lower FSH threshold. Therefore, a lower dose of exogenous FSH can attain sufficient ovarian stimulation. The two most commonly used approaches for ovulation induction with gonadotropins in clinical practice are the low-dose step-up and the low-dose step-down protocols. The chronic low dose protocols will reduce the risk of multiple follicular developments and their associated perils, including multiple pregnancies and OHSS. Additionally, the gonadotropin treatment is quite expensive, timeconsuming and requires continuous monitoring by expert [35].

\section{Glucocorticoids}

Glucocorticoids are a class of corticosteroids having steroidal skeleton and secreted from the adrenal glands in stress response and effective at reducing inflammation and suppressing the immune 
system [36]. Glucocorticoids such as prednisone and dexamethasone (Figure 3) have been utilized in the anovulation treatment. In PCOS patients resistant to clomiphene treatment and having normal androgen levels, the addition of dexamethasone in high dose and short duration to clomiphene displayed a favorable response for induction of ovulation with minimal anti-estrogenic effect on the endometrium and higher ovulation and pregnancy rates. In PCOS patients with elevated androgen levels, only the low dexamethasone dose was efficient in increasing ovulation and pregnancy rate. Additionally, in PCOS patients, the addition of glucocorticoids during induction of ovulation by letrozole showed substantial improvement in folliculogenesis, ovulation, and pregnancy. However, glucocorticoids usage is associated with potentially adverse effects on insulin sensitivity; hence, its prolonged use must be avoided [37].

\section{Laparoscopic Ovarian Diathermy}

Laparoscopic ovarian drilling with laser or diathermy is a surgical treatment used to destroy part of the ovary in PCOS patients with anovulation [38]. It is recommended as a secondline treatment in clomiphene-resistant PCOS women who cannot undergo gonadotropin treatment because of high cost and continuous monitoring. In clomiphene-resistant PCOS women undergoing LOD versus gonadotropin treatment, the pregnancy rate was lower, whereas no difference in ovulation or pregnancy, live birth, and miscarriage rate was observed. In addition, ovarian drilling was ineffective in improving metabolic abnormalities, and in some PCOS patients, it exhibited temporary fertility advantages and required adjuvant therapy after drilling with clomiphene. LOD was more effective in patients with high LH levels, and after surgery, a substantial decrease in $\mathrm{LH}$ and androgen levels was observed. Furthermore, in some women (63\%-85\%), LOD retained the menstrual cycle and caused a prolonged positive effect on the reproductive system [39].

\section{In vitro Fertilization}

In Vitro Fertilization (IVF) is an assistive reproductive technology that involves fertilizing an egg with sperm "in vitro." After the fertilized egg experiences embryo culture, it is stored or implanted in the uterus for creating a successful pregnancy. The in vitro fertilization technique is primarily used in patients where other treatments, including clomiphene citrate, letrozole, gonadotropins, etc., were unsuccessful.
It is one of the last options to attain pregnancy in PCOS women [40]. However, in some patients with concomitant diseases, IVF is the first option. IVF in PCOS women have displayed decent pregnancy and live birth rates with reduced risk of multiple pregnancies. Therefore it is one of the rational options to accomplish pregnancy in PCOS women. However, the IVF procedure is expensive with no guarantee of success and can take patients' emotional/psychological toll. Additionally, other drawbacks of in vitro fertilization include higher chances of ovarian hyper-stimulation syndrome (OHSS), multiple pregnancies (around 20-30\%), ectopic pregnancy, and risk of prematurity and low birth weight in babies [41].

\section{Therapy for Insulin Resistance in PCOS Women}

The occurrence of insulin resistance measured by compromised glucose tolerance and its incidence rate is much higher in PCOS women than women with no PCOS and the same age and weight. Insulin resistance is associated with diabetes, metabolic syndrome, and in later stages with cardiovascular issues [42]. The treatment of PCOS with diabetes needs proper lifestyle changes including, diet and exercise and proper medications. The most common medicine to improve insulin resistance in PCOS patients is metformin (Figure 4) [43]. Metformin is an oral antidiabetic drug that belongs to the biguanide class, sold under the brand name Glucophage to treat type 2 diabetes mellitus. It is used as a first-line drug for treating type 2 diabetes, particularly in obese patients; however, it works more effectively in non-obese PCOS women than obese PCOS patients. It is used as a second-line agent to treat infertility in patients suffering from polycystic ovary syndrome. It is related to an increase in the menstrual cycle, improvement in ovulation, and reduction in circulating androgen level. In addition, metformin can support weight loss by enhancing metabolic functions [44]. Metformin's clinical role is to obstruct glucose production, reduce glucose uptake by the intestine and increase insulin sensitivity in peripheral tissues. In PCOS women, metformin improves ovulation induction by lowering insulin levels and varying the insulin effect on ovarian androgen biosynthesis, theca cell proliferation, and endometrial growth [45]. Moreover, it can inhibit ovarian gluconeogenesis and therefore lowering ovarian androgen production. Metformin's safe profile makes it the most commonly used drug in treating glucose intolerance and elevated diabetes risk in PCOS women. Metformin

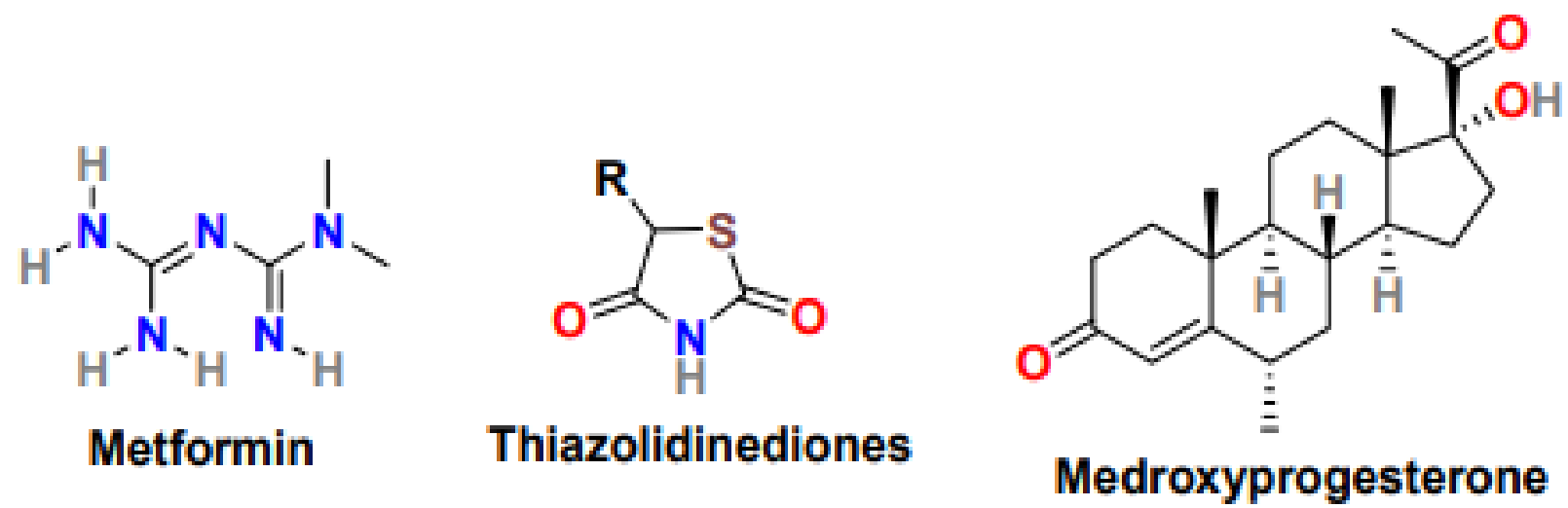

Figure 4: Chemical structure of drugs for the treatment of insulin resistance in PCOS women. 
is given in numerous routines with focus and monitoring on patient tolerance. The target dose for metformin is $1500-2550 \mathrm{mg} /$ day, which is achieved slowly by beginning with $500 \mathrm{mg} /$ day metformin for one week followed by $1000 \mathrm{mg} /$ day for another week and then $1500 \mathrm{mg} /$ day. Primarily the metformin exhibits response at the dose of $1000 \mathrm{mg} /$ day only in some patients the dose reaches 1500 or $2000 \mathrm{mg} /$ day. Metformin also works efficiently in combination with clomiphene and gonadotropins. Clomiphene can improve ovulation and pregnancy rate, whereas gonadotropins encourage mono ovulation and decrease the dosage and duration of gonadotropins and the risk of canceled cycles. The most common complications associated with metformin use are nausea, diarrhea, weakness, flatulence, myalgia, hypoglycemia, and abdominal pain. Thiazolidinediones (Figure 4), also known as glitazones, are another class of insulin-sensitizing drug that has displayed good ovulation and pregnancy rates [46]. These drugs improve insulin sensitivity by increasing the ovulation rate and glucose tolerance and reducing circulating androgen. Troglitazone, rosiglitazone, and pioglitazone molecules of the thiazolidinediones class have been studied extensively, however in animal studies; these molecules possess hepatotoxicity, cardiovascular risk, weight gain, and reproductive toxicity and therefore resulted in restricted use in PCOS women [47].

\section{Therapy for Menstrual Dysfunction in PCOS Women}

PCOS patients mainly suffer from abnormal menstrual patterns because of prolonged anovulation, and these menstrual irregularities usually have a history dating back to menarche [48]. Some PCOS women have oligomenorrhea, menstrual bleeding $<9$ menstrual periods per year, or secondary amenorrhea, which is the absence of menstruation for six months. In addition, other consequences of anovulatory menstrual cycles include dysfunctional uterine bleeding and infertility. Ongoing anovulation can increase the risk of endometrial hyperplasia and carcinoma. Therefore, it must be treated on time. The most common approach to treat menstrual abnormalities in PCOS women is oral contraceptives [49]. In PCOS women who do not wish for pregnancy, hormonal contraceptives are the first-line treatment for menstrual irregularities. PCOS women are administered using progestin such as medroxyprogesterone (Figure 4) or oral contraceptive with a combination of estrogen and progestin to reduce circulating androgens, maintain regular menstrual cycles, and reduce the risk of endometrial hyperplasia and cancer. Additionally, metformin is used to improve insulin resistance and menstrual irregularities in PCOS women who cannot take or tolerate hormonal contraceptives or lifestyle changes that have no positive effect on them. Metformin can reduce free testosterone levels, cure metabolic and glycemic abnormalities and maintain regular menstrual cycles.

\section{Therapy for Hyperandrogenism Related Symptoms in PCOS Women}

Hyperandrogenism is one of the principal features of PCOS and is clinically displayed as hirsutism, acne, and alopecia, etc. [50]. The hyperandrogenism symptoms vary from patient to patient, and subsequently, its treatment.

\section{Hirsutism}

Hirsutism is a condition in women that results in excessive terminal hair in androgen-dependent areas of the body. This disorder mainly occurs because of increased androgen action on hair follicles or upsurge circulating level of androgens or amplified sensitivity of hair follicles to normal levels of circulating androgens. Treatment options for hirsutism in women with PCOS or without PCOS are the same. They include therapies that aim to local expressions of hirsutism or target underlying causes using proper medications [51]. The effective therapies that target the local expressions of hirsutism include hair removal using physical means such as shaving, laser therapy, electrolysis, topical treatment, etc. Pharmacological treatment is focused on blocking the androgen action at hair follicles or restricting androgen production [52].

Medications under pharmacological treatment generally take around six months to show a significant effect on hair growth. Patients intolerant to medical therapy are treated with a combination of local measures and drug treatment. Several medicines have been studied for the treatment of hirsutism in PCOS patients, and the most effective drug options are oral contraceptives, antiandrogens, and topical cream. Low-dose Oral Contraceptive Pills (OCPs) are mainly used in women who do not wish to conceive. Birth control pills or oral contraceptives comprising estrogen and progestin are used to treat hirsutism caused by androgen production; however, the selection of oral contraceptives is substantial because some of the progestins also have an androgenic effect. The low-dose OCPs available in the market include ethinyl estradiol (Figure 5) in doses ranging from 15-35g. OCPs with less androgenic progestin such as norgestimate, gestodene, and desogestrel (Figure 5) are good options for hirsutism treatment [53]. Anti-androgens, a drug that blocks androgens from binding to the receptor, are prescribed after six months of ineffective oral contraceptive treatment. Response to antiandrogen in hirsutism treatment is prolonged and sometimes takes around 18 months, and the most commonly used antiandrogens are spironolactone (Aldactone) and flutamide (Eulexin) (Figure 5). Spironolactone is safe and low- cost drug that possesses moderate antiandrogenic effects when monitored in high doses (100-200 mg daily). It works as dose-dependent competitive inhibitors of the androgen receptor and demonstrates effectiveness on hirsutism treatment. Flutamide is non-steroidal, selective antiandrogen with no progestogenic effect and equally effective as spironolactone, but its application required hepatic function monitoring. In addition, estrogen-progestin combination therapy, including a combination of OCPs, effectively reduces terminal hair growth and acne formation in PCOS patients.

Moreover, topical cream naming Eflornithine (Vaniqa) (Figure 5) is a prescription cream that displayed effectiveness in slowing down the growth rate of excessive facial hair in women [54]. Eflornithine is ineffectual in completely removing the existing hair. Therefore, it is used in combination with laser therapy to enhance its response. Gonadotropin-releasing hormone agonist (Gn-RHa) including, leuprolide (Lupron), is effective in women with severe insulin resistance who are unresponsive to combination hormonal therapy or not able to tolerate oral contraceptive pills [55]. It works 
(N)

Ethinyl estradiol<smiles>C#C[C@]1(O)CC[C@H]2[C@H]3CCC4=CCCC[C@H]4[C@H]3C(=C)C[C@]21CC</smiles>

Desogestrel<smiles>C=C[C@@]1(OC(C)=O)CC[C@H]2[C@H]3CCC4=C/C(=N/O)CC[C@H]4[C@H]3CC[C@]21CC</smiles>

Norgestimate

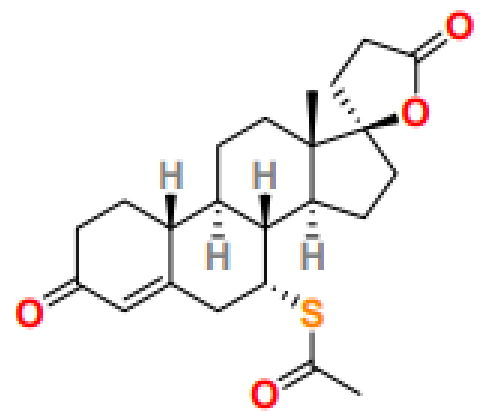

Spironolactone

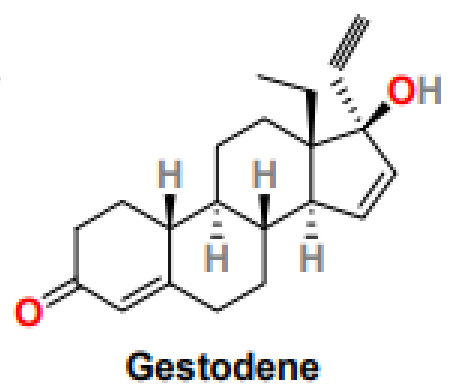<smiles>CC(C)C(=O)Nc1ccc([N+](=O)[O-])c(C(F)(F)F)c1</smiles>

Flutamide<smiles>CNC(CCCNN)(C(=O)CO)C(F)F</smiles>

Eflornithine

Figure 5: Chemical structure of drugs for the treatment of hyperandrogenism-related symptoms inPCOS women.

by suppressing pituitary hormones, reducing androgen and estradiol secretion, and subsequently reducing the severity of hirsutism; however, the Gn-RHa treatment is expensive, and its use is associated with long-term consequences as hot flushes, bone demineralization, atrophic vaginitis. In some PCOS women with elevated adrenal androgen levels, glucocorticoids suppress adrenal androgen secretion [56]. Glucocorticoids such as prednisone and dexamethasone have shown efficiency against hirsutism in patients with classic congenital adrenal hyperplasia and retained normal ovulatory cycles. In addition, insulin-lowering agents are beneficial in patients struggling with terminal hair growth. Metformin and thiazolidinediones have shown effectiveness in lowering ovarian androgen secretion by improving insulin sensitivity. Metformin therapy has shown improvement in clinical manifestations of hyperandrogenism [57].

\section{Acne and Alopecia}

For acne treatment, both oral contraceptive pills and antiandrogens have been used successfully, whereas for alopecia, there are no extensive trials, but oral contraceptive pills and antiandrogens are administered [58,59]. Oral contraceptive pills treatment has shown a reduction in inflammatory acne count around $30-60 \%$ and is very useful in patients with deep-seated nodules or relapsing on isotretinoin. For alopecia treatment, spironolactone has shown some effect in few studies similar to finasteride.

\section{PCOS Treatment with Natural Products}

Natural products have been in immense use throughout human evolution. Several natural products from plants are used to cure various types of diseases in humans. Correspondingly, the natural molecules affecting the various pathological aspects of PCOS play a significant role in overcoming PCOS-related symptoms [60].

\section{Inositol}

Inositol is a vitamin-like substance with a basic chemical formula similar to glucose (C6H12O6) and is present in many plants and animals. Inositol exists in different stereoisomers with Myo-inositol (Myo-Ins) and D-chiro-inositol (D-Chiro-Ins) (Figure 6) the most common [61]. Both the isomers are the second messenger of insulin. The Myo-Ins (expression of glucose transporters and cellular glucose uptake) and D-Chiro-Ins (glycogen synthesis and storage) are involved in different functions. Furthermore, physiologically the Myo-Ins is converted into D-Chiro-Ins through the activation of the insulin-dependent epimerase enzyme. PCOS women mostly exhibit compromised inositol metabolism and insulin resistance, causing a reduction in the intracellular conversion of Myo-Ins to D-Chiro-Ins inositol [62]. Several studies suggested that the dietary supplementation of Myo- Ins, alone in combination with D-ChiroIns, effectively improves metabolic and hormonal profile, reduces hyperandrogenism, refining oocyte quality, and maintains a regular menstrual cycle. Mainly, in obese patients, the 40:1 ratio of MyoIns and D-Chiro-Ins was most effective in restoring ovulation and normalizing the progesterone, LH, SHBG, estradiol, and testosterone level [63]. Inositol can efficiently regulate glucose metabolism, and therefore in PCOS treatment, it can utilize competently.

\section{Flavonoids}

Naringenin (Figure 6) is a tasteless and colorless flavanone present primarily in grapefruit and in various fruits and herbs [64]. Studies with naringenin in PCOS women suggest that it can decrease the level of testosterone and estradiol and increase the concentration of enzymes involved in scavenging reactive oxygen specie. Additionally, naringenin exhibited positive cytoprotective and anti-inflammatory results in the animal model, inhibited PCOS-associated weight 
<smiles>O[C@H]1[C@H](O)[C@H](O)[C@H](O)[C@@H](O)[C@H]1O</smiles>

Myo-inositol<smiles>O[C@H]1[C@H](O)[C@H](O)[C@H](O)[C@@H](O)[C@H]1O</smiles>

D-chiro-inositol<smiles>O=C1C[C@H](c2ccc(O)cc2)Oc2cc(O)cc(O)c21</smiles>

Naringenin<smiles>O=C1O[C@H]([C@@H](O)CO)C(O)=C1O</smiles>

Vitamin C<smiles>C[C@H]1O[C@H](OC[C@H]2O[C@H](Oc3c(-c4ccc(O)c(O)c4)oc4cc(O)cc(O)c4c3=O)[C@H](O)[C@@H](O)[C@@H]2O)[C@H](O)[C@@H](O)[C@@H]1O</smiles><smiles>Cc1c(C)c2c(c(C)c1O)CC[C@@](C)(CCC[C@H](C)CCC[C@H](C)CCCC(C)C)O2</smiles><smiles>CC/C=C/C/C=C/C/C=C/CCCCCCCC(=O)O</smiles>

Apha-linolenic acid

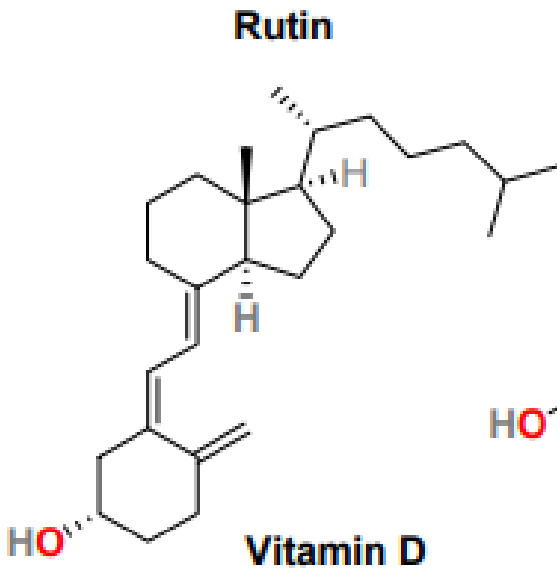<smiles>CCC=CCC=CCC=CCC=CCC=CCCCC(=O)O</smiles>

Eicosapentaenoic acid<smiles>CC/C=C\C/C=C\C/C=C\C/C=C\C/C=C/C/C=C/CCC(=O)O</smiles>

\section{Docosahexaenoic acid}

Figure 6: The chemical structure of natural products is effective in the treatment of PCOS.

gain, and reduced serum glucose levels [64]. Another flavonoid that positively affects PCOS treatment is rutin (Figure 6), a plant pigment found in certain fruits and vegetables. In obese mice, rutin can control obesity and insulin resistance, and rutin treatment is significantly effective against hyperandrogenism and infertility.

\section{Vitamins}

Vitamin C (Figure 6), also called ascorbic acid, is a micronutrient essential for cells and tissues' physiological and healthy growth. It is a watersoluble vitamin with antioxidant properties and can restore fat-soluble vitamin E (Figure 6) antioxidant nature. In PCOS rats, it was observed that vitamin $\mathrm{C}$ level was controlled throughout the menstrual cycle. It plays a significant role in regulating the menstrual cycle and ovarian functions [65]. Vitamin E, also known as tocopherol, is a fat-soluble vitamin with antioxidant properties, and it neutralizes free radicals and promotes cell renewal. Because of its anticoagulant and antioxidant properties, Vitamin
E displays the ability to improve endometrial thickness in women with idiopathic infertility. It effectively reduced oxidative stress and subsequently reduced the exogenous human menopausal gonadotropin; however, its intake does not affect the pregnancy rate [66]. Vitamin D (Figure 6) a fat-soluble secosteroid essential for calcium homeostasis and bone mineralization. In humans, vitamin D3, known as cholecalciferol and vitamin D2, also called ergocalciferol, are the most common forms of Vitamin D. Recent reports suggest that vitamin D deficiency plays a part in insulin resistance and inflammation, dyslipidemia, and infertility in PCOS women. Therefore, Vitamin D as a supplement may reduce insulin resistance and hyperandrogenism in patients with PCOS [67]. In addition, it was observed that the average vitamin $\mathrm{D}$ level in women helps attain more endometrium thickness and, therefore, increases the chances of pregnancy. It is evident that vitamins affect the various pathological features of PCOS; therefore, further study is required to establish the positive impact of vitamins in PCOS treatment. 


\section{Omega-3 Fatty Acids}

Omega-3 fatty acids are polyunsaturated fatty acids, and their three primary forms are Alpha- Linolenic Acid (ALA), Eicosapentaenoic Acid (EPA), and Docosahexaenoic Acid (DHA). The ALA is found in plant oil such as flaxseed, soya bean, and canola oil. The biologically active Eicosapentaenoic Acid (EPA) and Docosahexaenoic Acid (DHA) are common in fish and other seafood. Omega-3 fatty acids are notable for their antioxidant, anti-inflammatory, anti-obesity, and insulin-sensitizing activity. Reports suggest that omega 3- fatty acids control insulin resistance and maintain total cholesterol, triglyceride, and low-density lipoprotein. Though no confirmation data is available suggesting the direct effect of omega- 3 fatty acids on BMI, fasting insulin and glucose, and $\mathrm{HDL}<\mathrm{FSH}, \mathrm{LH}, \mathrm{SHGB}$, and total testosterone. In PCOS women, Omega-3 fatty acid supplements may decrease inflammation because of a reduction in high-sensitivity C-reactive protein and an increase in adiponectin level [68]. It's recommended to administer omega-3-fatty acids in PCOS women with inflammatory and cardiovascular-related symptoms [69]. The most common side effects of omega-3-fatty acids include mild gastrointestinal discomfort, intestinal gas, nausea, diarrhea, headache, and synergistic effects. It is not recommended during antiplatelet and anticoagulant treatment, and its use requires continuous monitoring in obese PCOS patients.

\section{Herbal Plants in PCOS Treatment}

At present, no perfect treatment is available for the PCOS symptoms. Therefore, herbal plants bearing active compounds are practical alternatives to available drugs and have attracted much attention in recent years [70]. The treatment of PCOS women with M. spicata (spearmint) tea twice a day exhibited a positive effect and decreased the level of free and total testosterone, increased the FSH and LH level, and subsequently decreased the hirsutism. The oral treatment with cinnamon (C. Zeylancam) can improve insulin sensitivity, and PCOS women can experience a significant decrease in insulin resistance. The Maitake mushroom (Hen-of-the-wood) extract co- treatment with clomiphene citrate significantly improved insulin sensitivity and supported ovulation in PCOS patients [71]. The O. Majorana (Sweet Marjoram) tea treatment can improve insulin sensitivity and act as an antiandrogen, subsequently decreasing fasting insulin levels and DHEA-S [72]. In combination with metformin, the fenugreek ( $T$. foenum graceum) seeds also show insulin sensitivity, reduce polycystic ovaries, and improve menstrual cycles [73]. Additionally, the plant has a large amount of phytoestrogen, including raspberry, licorice, soya been, etc. are having antiandrogenic properties and are therefore effective in decreasing androgen levels in PCOS patients [74]. The plants having an antioxidant property such as C. Sinensis (Green tea), B. Vulgaria (bamboo), P. granatum I. (Pomegranate juice), etc. significantly improve serum level of sex hormone and reduce oxidative stress [75].

\section{Conclusion}

PCOS is a severe heterogeneous disorder found in women with no complete understanding of its pathophysiology. No permanent therapy is available for PCOS. However, its treatment is symptombased. PCOS-associated symptoms include anovulation, insulin resistance, menstrual dysfunction, hirsutism, hyperandrogenism, acne, and alopecia. PCOS women are susceptible to obesity, diabetes, and adverse cardiotoxicity. The review article provides comprehensive information regarding the most common therapies advantageous in treating PCOS symptoms. Several approved drugs applicable in the treatment of various PCOS symptoms have been discussed in detail. In addition, the natural products and herbal plants exhibiting beneficial effects in the preliminary examination on PCOS women have been included in the article. Substantial work is required to understand the pathophysiology and genetics of PCOS syndrome to develop specific treatment and to delay the long-term effects of PCOS in women. Furthermore, extensive research is needed to investigate more drugs like chemical compounds and natural products to discover a suitable cure for improving PCOS symptoms. The information presented here is beneficial to researchers, clinicians, and the pharmaceutical industry and promotes finding and developing PCOS treatment.

\section{References}

1. Ndefo UA, Eaton A, Green MR (2013) Polycystic ovary syndrome: a review of treatment options with a focus on pharmacological approaches. Physical Therapy 38: 336-355. [crossref]

2. El Hayek S, Bitar L, Hamdar LH, Mirza FG, Daoud G (2016) Poly Cystic Ovarian Syndrome: An Updated Overview. Front Physiol 7: 124. [crossref]

3. Gambineri A, Patton L, Altieri P, Pagotto U, Pizzi C, et al. (2012) Polycystic ovary syndrome is a risk factor for type 2 diabetes: results from a long-term prospective study. Diabetes 61: 2369-2374. [crossref]

4. Kshetrimayum C, Sharma A, Mishra VV, Kumar S (2019) Polycystic ovarian syndrome: Environmental/occupational, lifestyle factors; an overview. J Turk Ger Gynecol Assoc 2: 255-263. [crossref]

5. Wawrzkiewicz-Jałowiecka A, Kowalczyk K, Trybek P, Jarosz T, Radosz P, et al. (2020) In Search of New Therapeutics-Molecular Aspects of the PCOS Pathophysiology: Genetics, Hormones, Metabolism and Beyond. Int J Mol Sci 21: 7054. [crossref]

6. Shagufta, Ahmad I (2021) The race to treat COVID-19: Potential therapeutic agents for the prevention and treatment of SARS-CoV-2. Eur. J. Med. Chem 213: 113-157. [crossref]

7. Shagufta, Ahmad I, Mathew S, Rehman S (2020) Recent progress in selective estrogen receptor down regulators (SERDs) for the treatment of breast cancer. RSC Medicinal Chemistry 11: 438-454. [crossref]

8. Shagufta, Ahmad I (2020) Transition metal complexes as proteasome inhibitors for cancer treatment. Inorganica Chimica Acta 506: 119-521.

9. Shagufta, Ahmad I (2018) Tamoxifen a pioneering drug: An update on the therapeutic potential of tamoxifen derivatives. European Journal of Medicinal Chemistry 143: 515531. [crossref]

10. Shagufta, Ahmad I (2017) An insight into the therapeutic potential of quinazoline derivatives as anticancer agents. Med Chem Comm 8: 871-885. [crossref]

11. Shagufta, Ahmad I, Panda G (2017) Quest for steroidomimetics: Amino acids derived steroidal and non-steroidal architectures. European Journal of Medicinal Chemistry 133: 139-151. [crossref]

12. Shagufta, Ahmad I (2016) Recent insight into the biological activities of synthetic xanthone derivatives. European Journal of Medicinal Chemistry 116: 267-280. [crossref]

13. Ahmad I, Shagufta (2015) Recent developments in steroidal and non-steroidal aromatase inhibitors for the chemoprevention of estrogen-dependent breast cancer. European Journal of Medicinal Chemistry 102: 375-386. [crossref]

14. Ahmad I, Shagufta (2015) Sulfones: An important class of organic compounds with diverse biological activities. International Journal of Pharmacy and Pharmaceutical Sciences 7: 19-27.

15. Harris HR, Titus LJ, Cramer DW, Terry KL (2017) Long and irregular menstrual cycles, polycystic ovary syndrome, and ovarian cancer risk in a population-based case-control study. Int J Cancer 140: 285-291. [crossref] 
16. Witchel SF, Oberfield SE, Peña AS (2019) Polycystic Ovary Syndrome: Pathophysiology, Presentation, and Treatment with Emphasis on Adolescent Girls. J Endocr Soc 3: 1545-1573. [crossref]

17. Amini L, Valian K, Avvalshahr HS, Montaeri A (2014) Self-Confidence in Women with and without Polycystic Ovary Syndrome. J Family Reprod Health 8: 113-116. [crossref]

18. Pasquali R, Pelusi C, Genghini S, Cacciari M, Gambineri A (2003) Obesity and reproductive disorders in women. Hum Reprod Update 9: 359-372. [crossref]

19. Sam S (2007) Obesity and Polycystic Ovary Syndrome. Obes Manag 3: 69-73. [crossref]

20. Zhang X, Zheng Y, Guo Y, Lai Z (2019) The Effect of Low Carbohydrate Diet on Polycystic Ovary Syndrome: A Meta-Analysis of Randomized Controlled Trials. Int J Endocrinol 2019: 4386401. [crossref]

21. Harrison CL, Lombard CB, Moran LJ, Teede HJ (2011) Exercise therapy in polycystic ovary syndrome: a systematic review. Human Reproduction Update 17: 171-183. [crossref]

22. Luo D, Yang Q, Zhou L, Wang H, Li F, et al. (2020) Comparative Effects of Three Kinds of Bariatric Surgery: A Randomized Case-Control Study in Obese Patients. Diabetes Therapy 11: 175-183. [crossref]

23. Lee R, Mathew CJ, Jose MT, Elshaikh AO, Shah L, et al. (2020) A Review of the Impact of Bariatric Surgery in Women with Polycystic Ovary Syndrome. Cureus 12: e10811. [crossref]

24. Franks S, Hardy K (2020) What causes anovulation in polycystic ovary syndrome? Current Opinion in Endocrine and Metabolic Research 12: 59-65.

25. Xu XL, Deng SL, Lian ZX, Yu K (2021) Estrogen Receptors in Polycystic Ovary Syndrome. Cells 10: 459. [crossref]

26. Legro RS, Barnhart HX, Schlaff WD, Carr BR, Diamond MP, et al. (2007) Clomiphene, metformin, or both for infertility in the polycystic ovary syndrome. N Engl J Med 356: 551-566. [crossref]

27. Wang L, Wen X, Lv S, Zhao J, Yang T, et al. (2019) Comparison of endometrial receptivity of clomiphene citrate versus letrozole in women with polycystic ovary syndrome: A randomized controlled study. Gynecological Endocrinology 35: 862-865. [crossref]

28. Kishk EA (2018) Comparison of tamoxifen and clomiphene citrate for induction of ovulation in cases with thin endometrium. Evidence Based Women's Health Journal 8: 288-292.

29. Aref NK, Ahmed WAS, Ahmed MR, Sedik WF (2019) A new look at low-dose aspirin: Co- administration with tamoxifen in ovulation induction in anovulatory PCOS women. Journal of Gynecology Obstetrics and Human Reproduction 48: 673675. [crossref]

30. Pavone ME, Bulun SE (2013) Clinical review: The use of aromatase inhibitors for ovulation induction and superovulation. J Clin Endocrinol Metab 98: 1838-1844. [crossref]

31. Carroll N, Palmer JR (2001) A comparison of intrauterine versus intracervical insemination in fertile single women. Fertil Steril 75: 656-660. [crossref]

32. El-Aziz MMA, Fouad MS, Ouf TF (2019) Short letrozole therapy vs extended (long) letrozole therapy for induction of ovulation in women with polycystic ovary syndrome. Egyptian Journal of Hospital Medicine 74: 1884-1890. [crossref]

33. Sastre ME, Prat MO, Checa MA, Carreras RC (2009) Current trends in the treatment of polycystic ovary syndrome with desire for children. Ther Clin Risk Manag 5: 353360. [crossref]

34. Homburg R, Hendriks ML, Konig TE, Anderson RA, Balen AH, et al. (2012) Clomifene citrate or low-dose FSH for thefirst-line treatment of infertile women with anovulation associated with polycystic ovary syndrome: aprospective randomized multinational study. Hum Reprod 27: 468-473. [crossref]

35. Ege S, Bademkıran MH, Peker N, Tahaoglu AE, Çaça FNH, et al. (2020) A comparison between a combination of letrozole and clomiphene citrate versus gonadotropins for ovulation induction in infertile patients with clomiphene citrate resistant polycystic ovary syndrome-A retrospective study. Ginekologia Polska 91: 185-188. [crossref]

36. Elnashar A, Abdelmageed E, Fayed M, Sharaf M (2006) Clomiphene citrate and dexamethazone in treatment of clomiphene citrate-resistant polycystic ovary syndrome: a prospective placebo- controlled study. Hum Reprod 21: 1805-1808. [crossref]
37. Suh S, Park MK (2017) Glucocorticoid-Induced Diabetes Mellitus: An Important but Overlooked Problem. Endocrinol Metab (Seoul) 32: 180-189. [crossref]

38. Mitra S, Nayak PK, Agrawal S (2015) Laparoscopic ovarian drilling: An alternative but not the ultimate in the management of polycystic ovary syndrome. J Nat Sci Biol Med 6: 40-48. [crossref]

39. Al-Fadhli R, Tulandi T (2004) Laparoscopic treatment of polycystic ovaries: is its place diminishing? Curr Opin Obstet Gynecol 16: 295-298. [crossref]

40. Tang K, Wu L, Luo Y, Gong B (2021) In vitro fertilization outcomes in women with polycystic ovary syndrome: A meta-analysis. European Journal of Obstetrics and Gynecology and Reproductive Biology 259: 146-152. [crossref]

41. Refaat B, Dalton E, Ledger WL (2015) Ectopic pregnancy secondary to in vitro fertilisation-embryo transfer: pathogenic mechanisms and management strategies. Reprod Biol Endocrinol 13: 30. [crossref]

42. Ehrmann DA (2005) Polycystic ovary syndrome. N Engl J Med 352: 1223-1236.

43. Onalan G, Goktolga U, Ceyhan T, Bagis T, Onalan R, et al. (2005) Predictive value of glucose-insulin ratio in PCOS and profile of women who will benefit from metformin therapy: obese, lean, hyper or normoinsulinemic? Eur J Obstet Gynecol Reprod Biol 123: 204-211. [crossref]

44. Yerevanian A, Soukas AA (2019) Metformin: Mechanisms in Human Obesity and Weight Loss. Curr Obes Rep 8: 156-164. [crossref]

45. Mahamed RR, Maganhin CC, Sasso GRS, de Jesus Simões M, Baracat MCP, et al. (2018) Metformin improves ovarian follicle dynamics by reducing theca cell proliferation and CYP-17 expression in an androgenized rat model. J Ovarian Res 11: 18. [crossref]

46. Rouzi AA, Ardawi MS (2006) A randomized controlled trial of the efficacy of rosiglitazone and clomiphene citrateversus metformin and clomiphene citrate in women with clomiphene citrate- resistant polycystic ovarysyndrome. Fertility and sterility 85: 428-435. [crossref]

47. Froment P, Touraine P (2006) Thiazolidinediones and Fertility in Polycystic Ovary Syndrome (PCOS). PPAR Res 2006: 73986. [crossref]

48. Ezeh U, Ezeh C, Pisarska MD, Azziz R (2021) Menstrual dysfunction in polycystic ovary syndrome: association with dynamic state insulin resistance rather than hyperandrogenism. Fertil Steril 115: 1557-1568. [crossref]

49. Shah D, Patil M (2018) National PCOS Working Group. Consensus Statement on the Use of Oral Contraceptive Pills in Polycystic Ovarian Syndrome Women in India. J Hum Reprod Sci 11: 96-118. [crossref]

50. Baptiste CG, Battista MC, Trottier A, Baillargeon JP (2010) Insulin and hyperandrogenism in women with polycystic ovary syndrome. J Steroid Biochem Mol Biol 122: 42-52. [crossref]

51. Spritzer PM, Barone CR, Oliveira FB (2016) Hirsutism in Polycystic Ovary Syndrome: Pathophysiology and Management. Curr Pharm Des 22: 5603-5613. [crossref]

52. Calaf J, López E, Millet A, Alcañiz J, Fortuny A, et al. (2007) Long-term efficacy and tolerability of flutamide combined with oral contraception in moderate to severe hirsutism: a 12-month, double-blind, parallel clinical trial. J Clin Endocrinol Metab 92: 3446-3452. [crossref]

53. Fraison E, Kostova E, Moran LJ, Bilal S, Ee CC, et al. (2020) Metformin versus the combined oral contraceptive pill for hirsutism, acne, and menstrual pattern in polycystic ovary syndrome. Cochrane Database Syst Rev 8: CD005552. [crossref]

54. Kumar A, Naguib YW, Shi YC, Cui Z (2016) A method to improve the efficacy of topical eflornithine hydrochloride cream. Drug Deliv 23: 1495-1501. [crossref]

55. Azziz R, Ochoa TM, Bradley EL, Potter HD, Boots LR (1995) Leuprolide and estrogen versus oral contraceptive pills for the treatment of hirsutism: a prospective randomized study. J Clin Endocrinol Metab 80: 3406-3411. [crossref]

56. Azziz R, Black V, Hines GA, Fox LM, Boots LR (1998) Adrenal androgen excess in the polycystic ovary syndrome: sensitivity and responsively of the hypothalamicpituitary-adrenal axis. J Clin Endocrinol Metab 83: 2317-2323. [crossref]

57. Sanoee MF, Neghab N, Rabiee S, Amiri I (2011) Metformin therapy decreases hyperandrogenism and ovarian volume in women with polycystic ovary syndrome. Iran J Med Sci 36: 90-95. [crossref]

58. Huber J, Walch K (2006) Treating acne with oral contraceptives: use of lower doses. Contraception 73: 23-29. [crossref] 
59. Shapiro J (2007) Clinical practice. Hair loss in women. N Engl J Med 357: 1620-1630. [crossref]

60. Iervolino M, Lepore E, Forte G, Laganà AS, Buzzaccarini G, et al. (2021) Natural Molecules in the Management of Polycystic Ovary Syndrome (PCOS): An Analytical Review. Nutrients 13: 1677. [crossref]

61. Unfer V, Facchinetti F, Orrù B, Giordani B, Nestler J (2017) Myo-inositol effects in women with PCOS: a meta-analysis of randomized controlled trials. Endocr Connect 6: 647-658. [crossref]

62. Laganà AS, Garzon S, Casarin J, Franchi M, Ghezzi F (2018) Inositol in polycystic ovary syndrome: Restoring fertility through a pathophysiology-based approach. Trends Endocrinol Metab 29: 768-780. [crossref]

63. Roseff S, Montenegro M (2020) Inositol treatment for PCOS should be science-based and not arbitrary. Int. J. Endocrinol 2020: 6461254. [crossref]

64. Kicinska A, Kampa RP, Daniluk J, Sek A, Jarmuszkiewicz W, et al. (2020) Regulation of the mitochondrial bk ca channel by the citrus flavonoid naringenin as a potential means of preventing cell damage. Molecules 25: 3010. [Crossref]

65. Olaniyan OT, Femi A, Iliya G, Ayobami D, Godam E, et al. (2019) Vitamin C suppresses ovarian pathophysiology in experimental polycystic ovarian syndrome. Pathophysiology 26: 331-341. [Crossref]

66. Chen J, Guo Q, Pei YH, Ren QL, Chi L, et al. (2020) Effect of a short-term vitamin E supplementation on oxidative stress in infertile PCOS women under ovulation induction: A retrospective cohort study. BMC Womens Health 20: 69. [Crossref]

67. Miao CY, Fang XJ, Chen Y, Zhang Q (2020) Effect of vitamin D supplementation on polycystic ovary syndrome: A meta-analysis. Exp. Ther. Med 19: 2641-2649. [Crossref]

68. Tosatti JAG, Alves MT, Cândido AL, Reis FM, Araújo VE, et al. (2021) Influence of $\mathrm{n}-3$ fatty acid supplementation on inflammatory and oxidative stress markers in patients with polycystic ovary syndrome: A systematic review and meta-analysis. $\mathrm{Br}$. J. Nutr 125: 657-668. [Crossref]

69. Wekker V, Van Dammen L, Koning A, Heida KY, Painter RC, et al. (2020) Long-term cardiometabolic disease risk in women with PCOS: A systematic review and metaanalysis. Hum Reprod 26: 942-960. [Crossref]

70. Kwon CY, Cho IH, Park KS (2020) Therapeutic Effects and Mechanisms of Herbal Medicines for Treating Polycystic Ovary Syndrome: A Review. Front Pharmacol 11: 1192. [Crossref]

71. Chen JT, Tominaga K, Sato Y, Anzai H, Matsuoka R (2010) Maitake mushroom (Grifola frondosa) extract induces ovulation in patients with polycystic ovary syndrome: a possible monotherapy and a combination therapy after failure with firstline clomiphene citrate. J Altern Complement Med 16: 1295-1299. [Crossref]

72. Haj-Husein I, Tukan S, Alkazaleh F (2016) The effect of marjoram (Origanum majorana) tea on the hormonal profile of women with polycystic ovary syndrome: a randomised controlled pilot study. J Human Nutrition Dietetics 29: 105-111. [Crossref]

73. Bashtian MH, Emami SA, Mousavifar N, Esmaily HA, Mahmoudi M, et al. (2013) Evaluation of Fenugreek (Trigonella foenum-graceum L.), Effects Seeds Extract on Insulin Resistance in Women with Polycystic Ovarian Syndrome. Iran J Pharm Res 12: 475-481. [Crossref]

74. Rajan RK, M SS, Balaji B (2017) Soy isoflavones exert beneficial effects on letrozoleinduced rat Polycystic Ovary Syndrome (PCOS) model through antiandrogenic mechanism. Pharm Biol 55: 242-251. [Crossref]

75. Soumya V, Muzib YI, Venkatesh P (2016) A novel method of extraction of bamboo seed oil (Bambusa bambos Druce) and its promising effect on metabolic symptoms of experimentally induced polycystic ovarian disease. Indian J Pharmacol 48: 162167. [Crossref]

\section{Citation:}

Shagufta, Ahmad I, Nasar NA, Imtiaz M (2022) Recent Approaches in the Treatment of Polycystic Ovary Syndrome: An Update. ARCH Women Health Care Volume 5(1): 1-11. 